\title{
Novel splice isoforms of pig myoneurin and their diverse mRNA expression patterns
}

\author{
Xiaohong Guo ${ }^{1, \text { a }}$, Meng $\mathrm{Li}^{1, \mathrm{a}}$, Pengfei Gao ${ }^{1}$, Guoqing Cao ${ }^{1}$, Zhimin Cheng', Wanfeng Zhang ${ }^{1}$, \\ Jianfeng $\mathrm{Liu}^{2}$, Xiaojun $\mathrm{Liu}^{3}$, and Bugao $\mathrm{Li}^{1{ }^{1, *}}$
}

\begin{abstract}
* Corresponding Author: Bugao Li Tel: +86-138-3540-1655, Fax: +86-0354-628-8228 E-mail: jinrenn@163.com

${ }^{1}$ College of Animal Science and Veterinary Medicine, Shanxi Agricultural University, Taigu, Shanxi 030801, China

${ }^{2}$ Key Laboratory of Animal Genetics Breeding and Reproduction, Ministry of Agriculture, College of Animal Science and Technology, China Agricultural University, Beijing 100193, China

${ }^{3}$ College of Animal Science and Veterinary Medicine, Henan Agricultural University, Zhengzhou, 450002, China
\end{abstract}

a These authors contributed equally to this work.

ORCID

Bugao Li

https://orcid.org/0000-0001-5130-7115

Submitted Dec 17, 2017; Revised Feb 21, 2018; Accepted Apr 23, 2018
Objective: The aim of this study was to clone alternative splicing isoforms of pig myoneurin $(M Y N N)$, predict the structure and function of coding protein, and study temporal and spatial expression characteristics of each transcript.

Methods: Alternative splice isoforms of MYNN were identified using RNA sequencing (RNAseq) and cloning techniques. Quantitative real-time polymerase chain reaction (qPCR) was employed to detect expression patterns in 11 tissues of Large White (LW) and Mashen (MS) pigs, and to study developmental expression patterns in cerebellum (CE), stomach (ST), and longissimus dorsi (LD).

Results: The results showed that MYNN had two alternatively spliced isoforms, MYNN-1 (GenBank accession number: KY470829) and MYNN-2 (GenBank accession number: KY670835). MYNN-1 coding sequence (CDS) is composed of 1,830 bp encoding $609 \mathrm{AA}$, whereas MYNN-2 CDS is composed of 1,746 bp encoding 581 AA. MYNN-2 was 84 bp less than MYNN-1 and lacked the sixth exon. MYNN-2 was found to have one $\mathrm{C}_{2} \mathrm{H}_{2}$ type zinc finger protein domain less than MYNN-1. Two variants were ubiquitously expressed in all pig tissues, and there were significant differences in expression of different tissues $(p<0.05$; $\mathrm{p}<0.01$ ). The expression of $M Y N N-1$ was significantly higher than that of $M Y N N-2$ in almost tissues ( $p<0.05 ; p<0.01$ ), which testified that $M Y N N-1$ is the main variant. The expression of two isoforms decreased gradually with increase of age in ST and CE of MS pig, whereas increased gradually in LW pig. In LD, the expression of two isoforms increased first and then decreased with increase of age in MS pig, and decreased gradually in LW pig.

Conclusion: Two transcripts of pig MYNN were successfully cloned and MYNN-1 was main variant. MYNN was highly expressed in ST, CE, and LD, and their expression was regular. We speculated that MYNN plays important roles in digestion/absorption and skeletal muscle growth, whereas the specific mechanisms require further elucidation.

Keywords: Pig; MYNN; Alternative Splicing; mRNA Expression

\section{INTRODUCTION}

The BTB (Broad complex, Tramtrack, Bricabrac) domain superfamily proteins have many functions such as activation and repression of transcription, cytoskeleton organization, and chromatin remodeling [1]. The BTB protein is not a traditional transcriptional regulator, as it functions as both a transcriptional activator and repressor [2-5]. It functions through rotation and reconstruction on the nucleosome to activate transcription [6]. It can also inhibit transcription factors (e.g., histone lysine-specific demethylase, promyelocytic leukemia zinc finger and B-Cell CLL/Lymphoma 6) [7-9] leading to inhibition of the transcriptional activity of related genes, mainly by the recruitment of transcriptional corepressors (e.g., nuclear receptor corepressor and its homolog SMRT, mSi3a [component of histone deacetylase com- 
plex], histone deacetylase) via its homodimeric domain [10-12]. The zinc finger protein is a kind of common transcriptional regulatory protein, which can regulate the expression of target genes mainly by combining with specific gene sequence, and combining with its own or other zinc finger protein. So then adapt to the development, differentiation and maturation to of organism at different stages of individual [13]. The study found that about $5 \%$ to $10 \%$ of $\mathrm{C}_{2} \mathrm{H}_{2}$ zinc finger proteins contain the BTB domain at the $\mathrm{N}$ end [14], which forms a new class of transcription factor $\mathrm{BTB} / \mathrm{POZ}$ and zinc finger proteins $(\mathrm{BTB} / \mathrm{POZ}-\mathrm{ZF})$ family, which usually act with other transcription factors or cofactors to form a transcriptional complex that activates or inhibits transcription. Myoneurin (MYNN) belongs to the BTB/POZ-ZF family, which contains an amino-terminal $\mathrm{BTB} / \mathrm{POZ}$ domain and eight tandem repeated zinc-finger motifs of the $\mathrm{C}_{2} \mathrm{H}_{2}$-type (zinc finger, $\mathrm{C}_{2} \mathrm{H}_{2}$ type) $[14,15]$, and plays an important role in the regulation of gene expression.

MYNN, identified in the mouse embryo, is highly homologous to the human protein [16]. Promoter beta-galactosidase constructs were used to define the region (first intron) that conferred basal expression of the MYNN gene [16]. The expression of MYNN was found to be abnormal during nerve crush or axotomy in extrasynaptic myonuclei, which proved that MYNN is a likely candidate to mediate electrical activitydependent expression of downstream synapse-specific genes [14]. MYNN was identified within the peripheral condensed chromatin and euchromatin/heterochromatin regions by electron microscopy analyses, and as such, may play an important role in chromatin structure and transcription [14]. MYNN can inhibit transcription in neuromuscular tissue, and its expression increased after denervation; therefore, it may regulate the expression of synaptic genes [14]. Studies have shown that MYNN represses gene transcription by recruiting a transcriptional corepressor and histone deacetylase [16]. MYNN expression in ovarian serous adenocarcinoma and lung (LU) squamous cell carcinoma was found to be as high as $34.1 \%$ and $33.3 \%$ respectively. In addition, MYNN is a potential promoting factor in head and neck squamous cell carcinoma, endometrial carcinoma, gastric carcinoma, and bladder epithelial cell carcinoma [17].

MYNN is widely expressed in various tissue types in mammals [18-20]. Northern blot analysis showed that in humans the expression of a major $2.5 \mathrm{~kb}$ MYNN in muscle was higher than that in other tissues including the testis, ovary, and placenta [15] The expression of MYNN was detected in the cerebellum $(\mathrm{CE})$, skeletal muscle, testis, heart $(\mathrm{H})$, brain, and liver (LI) tissues in the mouse [15]. Muscle tissue is a primary site of MYNN gene transcription, and the expression of nuclear $M Y N N$ is associated with subsynaptic nuclei at the neuromuscular junction, and is also developmentally regulated during the late embryonic period (E16, P0) [14]. By analyzing the overall structure of MYNN and examining its expression in human muscle, Alliel et al [15] showed that MYNN can regulate gene expression in the muscle. To date, there are no study on the expression patterns of MYNN in pig, especially its alternative splice variants.

The Mashen (MS) pig is a local breed in North China. It is hardy, adaptable to mountainous environments, and has good meat quality. However, the growth rate and lean/fat ratio are much lower than those in Western commercial pig breeds such as Large White (LW) and Landrace [21-23]. Previous studies have demonstrated the function of MYNN in the human and mouse, but no data have been reported about pig MYNN. The aim of this study was to identify the alternatively spliced transcript variants of pig MYNN using RNA sequencing (RNA-seq) results, and to measure their mRNA expression profiles to provide a foundation for further studies on this protein.

\section{MATERIALS AND METHODS}

All of the animal procedures were as per the Code of Ethics of the World Medical Association (Declaration of Helsinki) for animal experiments (http://ec.europa.eu/environment/ chemicals/lab_animals/legislation_en.htm). The methods were performed in accordance with the Good Experimental Practices adopted by the College of Animal Science and Veterinary Medicine, Shanxi Agricultural University (Shanxi, China). Moreover, the local animal welfare laws, guidelines, and policies were strictly followed for the feed and use of experimental animals.

\section{Animal and sample collection}

In this study, a total of nine healthy LW and nine healthy MS male pigs were selected from the Datong Pig Breeding Farm (Shanxi, China). All of the animals were kept under the same feeding and environmental conditions. They were weaned at 28 days old and were castrated when weaning. Three pigs of each breed were slaughtered at each of the three development stages at 1, 90, and 180 days after birth. Eleven different tissues including H, LI, spleen (SP), LU, kidney (K), CE, small intestine $(\mathrm{SI})$, stomach $(\mathrm{ST})$, pancrease $(\mathrm{P})$, longissimus dorsi $(\mathrm{LD})$, and fat $(\mathrm{F})$ were collected, immediately snapped in liquid nitrogen, and stored at $-80^{\circ} \mathrm{C}$ for subsequent use. The transcriptome of $\mathrm{LD}$ at each of the three development stages was subjected to RNA-seq analysis.

\section{RNA extraction and purification}

Total RNA was extracted using TRIZOL reagent (Life Technologies, Carlsbad, CA, USA) following the manufacturer's instructions. RNA integrity number (RIN) was checked to determine RNA integrity using an Agilent Bioanalyzer 2100 (Agilent Technologies, Santa Clara, CA, USA). All of the so- 
lutions had a RIN $\geq 7.0$ and $28 \mathrm{~S} / 18 \mathrm{~S} \geq 0.7$. Total RNA was further purified using the RNeasy Micro Kit (QIAGEN GmBH, Hilden, Germany) and RNase-Free DNase Set (QIAGEN, Germany).

\section{Sequencing and comparative analysis}

RNA $(2 \mu \mathrm{g})$ was depleted of ribosomal RNA (Beckman Coulter, Beverly, MA, USA). First strand cDNA was synthesized using SuperScript II Reverse Transcriptase, and second strand cDNA synthesis was subsequently performed using DNA polymerase I and RNase $\mathrm{H}$. The library fragments were purified with AMPure XP system (Beckman Coulter, USA). Thereafter, $2.5 \mu \mathrm{L}$ USER Enzyme (NEB, Ipswich, MA, USA) was added to size-selected, adaptor-ligated cDNA, and the mixture was incubated at $37^{\circ} \mathrm{C}$ for $15 \mathrm{~min}$ followed by $5 \mathrm{~min}$ at $95^{\circ} \mathrm{C}$. Subsequently, polymerase chain reaction (PCR) was performed with Phusion High-Fidelity DNA polymerase, Universal PCR primers, and Index (X) primer. The PCR products were purified (AMPure XP system; Beckman Coulter, USA) and the library quality was assessed on the Agilent Bioanalyzer 2100 system. The clustering of the index-coded samples was performed on a cBot Cluster Generation System using TruSeq PE Cluster Kit v4-cBot-HS (Illumina, San Diego, CA, USA) according to the manufacturer's instructions. After cluster generation, the library preparations were sequenced on an Illumina Hiseq 2500 platform to generate $2 \times 100$ base pair (bp) paired-end reads.

Raw data (raw reads) in fasta format were first processed through in-house perl scripts. In this step, clean data (clean reads) were obtained by removing the reads containing the adapter sequence, reads containing poly- $\mathrm{N}$, and low-quality reads from raw data. At the same time, Q20, Q30, GC content, and sequence duplication level of the clean data were calculated. All of the downstream analyses were based on clean data of high quality. Then these clean reads were mapped to the pig genome (UCSC susScr3, from the UCSC Genome Browser [ftp://hgdownload.cse.ucsc.edu/goldenPath/susScr3/bigZips/ susScr3.fa.gz]). Only the reads with a perfect match or one mismatch were further analyzed and annotated based on the reference genome. TopHat2 software was used to map the reference genome $[24,25]$.

\section{Prediction of alternative splicing}

Alternative splicing with less than $5 \%$ reads were filtered out. Then the alternative splicing sites were mapped to known splicing sites (1 bp error was allowed) to identify known alternative splicing sites. Finally, novel unmapped alternative splicing sites were classified.

Identification of pig MYNN alternative splice variants Based on the results of alternative splicing in RNA-seq, the primers in this experiment were designed using the NCBI Nucleotide System and then tested using Oligo6 software. Two primers were designed to amplify the distinctive variants and two primers were used for quantitative real-time PCR (qPCR), as shown in Table 1. The strategy is shown in Figure 1a. PCR reactions were performed in a $25 \mu \mathrm{L}$ reaction volume containing 50 ng cDNA Pool (18 muscle tissues were used for sequencing), $0.5 \mu \mathrm{M}$ of each primer, $12.5 \mu \mathrm{L}$ of $2 \times \mathrm{Taq} \mathrm{PCR}$ MasterMix (TIANGEN, Beijing, China), and $\mathrm{dd}_{2} \mathrm{O}$ to a total volume $25 \mu \mathrm{L}$. The PCR reaction conditions were as follows: denaturation at $94^{\circ} \mathrm{C}$ for $5 \mathrm{~min}$ followed by 35 cycles at $94^{\circ} \mathrm{C}$ for $30 \mathrm{~s}, 60^{\circ} \mathrm{C}$ for $30 \mathrm{~s}$, and $72^{\circ} \mathrm{C}$ for $30 \mathrm{~s}$, with a final extension at $72^{\circ} \mathrm{C}$ for $5 \mathrm{~min}$. The PCR products were examined by electrophoresis on a $1.0 \%$ agarose gel.

\section{Cloning of MYNN alternative splice variants and bioinformatics analysis}

PCR products were purified using a Gel Extraction Kit (Sangon Biotech, Shanghai, China) and ligated into the pMD19-T Vector (Takara, Dalian, China) for sequencing. Nucleic acid sequences were aligned using the NCBI Nucleotide Blast database (http://www.ncbi.nlm.nih.gov/blast) and DNAMAN 7 software. MegAlign software was used to align the amino acids of MYNN among 10 species to calculate their homology. Phylogenetic trees were constructed based on the neighborjoining method in MEGA4 and NCBI pairwise alignments

Table 1. PCR primer sequences of the pig MYNN gene for amplification

\begin{tabular}{|c|c|c|c|c|}
\hline Names & Primer sequences $\left(5^{\prime} \rightarrow 3^{\prime}\right)$ & Product sizes (bp) & Annealing $\left(\mathrm{Tm}^{\circ}{ }^{\circ} \mathrm{C}\right)$ & Notes \\
\hline \multirow[t]{2}{*}{ P1 } & F1:GTGAGAAGCCATACAAATGTGAA & 426 & 60 & Clone \\
\hline & R1:CAGAATGGACTTTTGTTTTGTGC & & & \\
\hline \multirow[t]{2}{*}{ P2 } & F2:AGAACAAGGGTAAAATTCGTTTGTG & 1928 & 60 & Clone \\
\hline & R2: TGCAGCATCAGGTGCTTTTA & & & \\
\hline \multirow[t]{2}{*}{ P3 } & F3: CCTCAGGAGAGCTCAACAAACA & 186 & 60 & qPCR for MYNN-1 \\
\hline & R3: TGGACTCTTTTTCACTCAAGGGAT & & & \\
\hline \multirow[t]{2}{*}{ P4 } & F4:CACTCATTCTCGAAAACATACAGGA & 170 & 60 & qPCR for MYNN-2 \\
\hline & R4:TGGACTCTTTTTCACTCAAGGGAT & & & \\
\hline \multirow[t]{2}{*}{185} & $\mathrm{~F}: C C C A C G G A A T C G A G A A A G A G$ & 132 & 60 & qPCR for $18 S$ rRNA \\
\hline & R:TTGACGGAAGGGCACCA & & & \\
\hline
\end{tabular}

$\mathrm{PCR}$, polymerase chain reaction; $M Y N N$, myoneurin; $\mathrm{PPCR}$, quantitative real-time $\mathrm{PCR}$. 
(a)

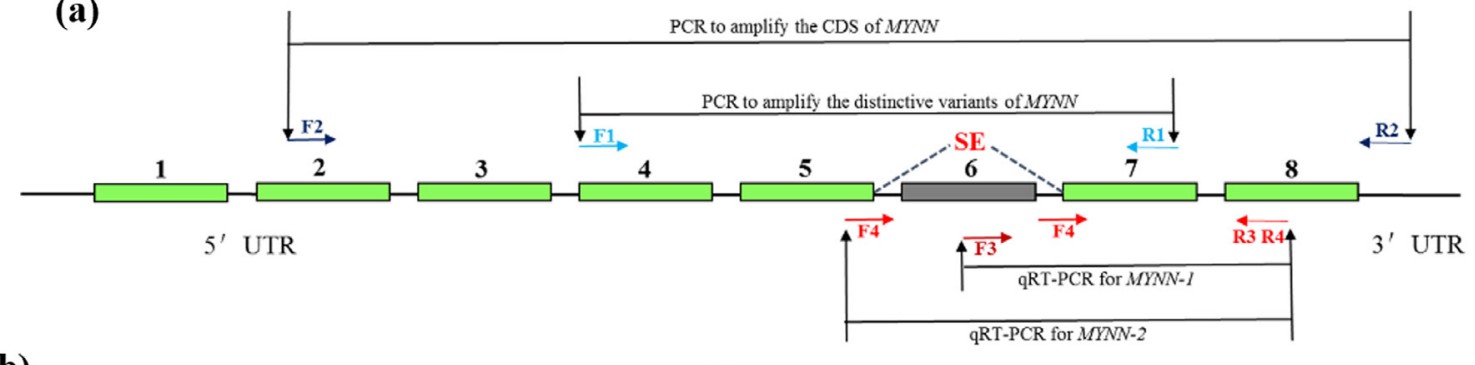

(b)

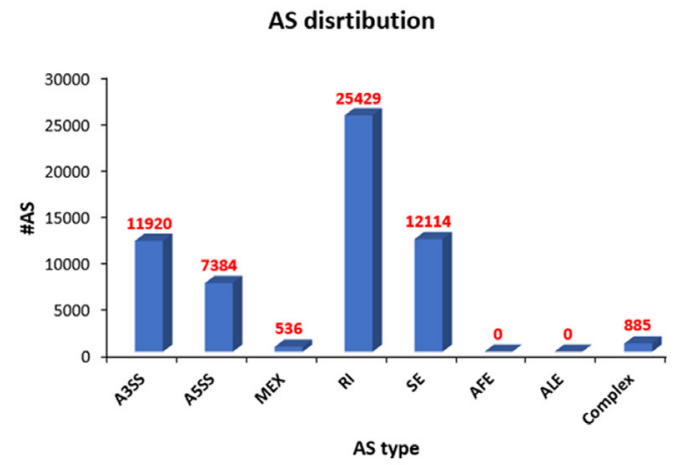

(c)

(c-1)

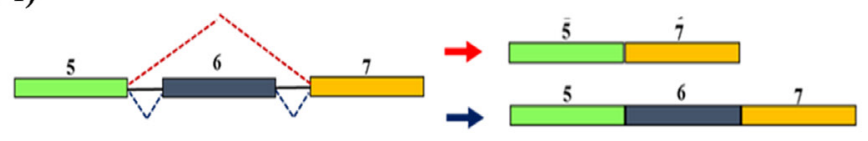

(c-2)

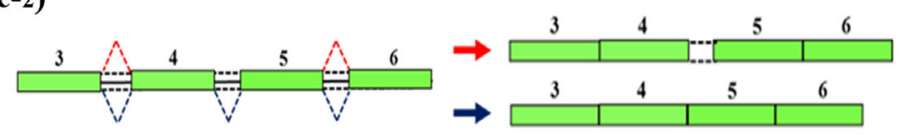

Figure 1. Genomic structure diagram of pig myoneurin (MYNN). (a) Cloning strategy for pig MYNN CDNA; (b) Classification of MYNN alternative splicing; (c) Genomic structure diagram of two alternative splice variants is predicted.

(https://blast.ncbi.nlm.nih.gov/Blast.cgi).

\section{Measurement of $M Y N N-1$ and $M Y N N-2$ mRNA expression levels}

To determine whether differentially expressed MYNN is involved in the development of muscle, we used qPCR to analyze expression of the two MYNN alternative splice variants in the LD of LW and MS pigs at three time points $(1 \mathrm{~d}, 90 \mathrm{~d}$, and 180 d). In addition to muscle, total RNA was extracted from 10 additional tissues of LW and MS pigs, including H, LI, SP, LU, $\mathrm{K}, \mathrm{CE}, \mathrm{SI}, \mathrm{ST}$, P, and F, at $90 \mathrm{~d}$. Specific primers for validation and spatial expression analysis of the MYNN alternative splice variants are shown in Table 1. Total RNA was extracted using Invitrogen Ambion TRIzol LS Reagent (Life Technologies, USA). The cDNA was synthesized by reverse transcription from 500 ng total RNA using the PrimeScript RT Reagent Kit with gDNA Eraser (Takara, China) according to the manufacturer's instructions. The qPCR was performed using a SYBR PrimeScript RT-PCR Kit (Takara, China) performed on an ABI-7500 (Life Technologies, USA) under the following conditions: pre-denaturation at $95^{\circ} \mathrm{C}$ for $30 \mathrm{~s}, 45$ cycles of $95^{\circ} \mathrm{C}$ for $5 \mathrm{~s}$ and $60^{\circ} \mathrm{C}$ for $34 \mathrm{~s}$, one cycle of $95^{\circ} \mathrm{C}$ for $15 \mathrm{~s}, 60^{\circ} \mathrm{C}$ for $1 \mathrm{~min}$, and $95^{\circ} \mathrm{C}$ for $30 \mathrm{~s}$. All of the qPCR reactions for each gene were performed using three biological replicates, with three replicates per experiment.

\section{Statistical analysis}

Average threshold $(\mathrm{Ct})$ values per triplicate were used to calculate the relative amounts of mRNA using the $2^{-\Delta \Delta \mathrm{Ct}}$ method, and $18 S$ rRNA was used as an internal gene. Statistical differences between the expression levels of MYNN splice variants were determined with analysis of variance (ANOVA) using SPSS 22.0. Differences between LW and MS pigs were analyzed using the Student's $t$-test, and differences among different time points were identified using ANOVA.

\section{RESULTS}

\section{RNA-seq results}

Illumina sequencing yielded approximately 2,718 million paired clean reads (100 nucleotides [nt]). The sequenced fragments were mapped to the pig genome in UCSC (susScr3) using TopHat software, which can align reads across splice junctions without relying on gene annotation. More than $73 \%$ of the total reads (2,793 million) were mapped to the pig genome. The results of TopHat included all alternative splicing information, which was classified as skipped exon (SE), alternative 5' splicing site, alternative 3 ' splicing site, retained intron (RI), mutually exclusive exon, alternative promoter, alternative polyadenylation. The results are shown in Figure $1 b$.

\section{Prediction of sites and types of alternative splice variants of $M Y N N$ in pig muscle}

Two types of MYNN alternative splice variants were predicted 
in pig muscle. An SE was predicted at the sixth exon (Figure 1c-1), and a RI was present at the fourth and fifth exons, which corresponded to the fourth intron (Figure 1c-2).

\section{Identification of alternative splice variants of pig MYNN in muscle tissue}

Two fragments of $510 \mathrm{bp}$ and $426 \mathrm{bp}$ were amplified by primer P1 (Figure 2a), and were named MYNN-1 and MYNN-2, respectively. Interestingly, after sequencing and alignment, we found that MYNN-2 lacked the sixth exon compared with MYNN-1 (Figure 2b). The splice sites complied with the GTAG rules at the 5' splice donor and 3' splice acceptor sites (Figure 2b).

Using a pool of cDNA as the template, the coding sequence (CDS) of $M Y N N-1$ and $M Y N N-2$ was amplified using P2 primers, leading to the successful amplification of two fragments of 2,012 bp and 1,928 bp (Figure 2c). The results of sequencing and alignment were in agreement with the alternative splice variants predicted by RNA-seq. MYNN-1 (GenBank accession number: KY470829) is 84 bp longer than MYNN-2 (GenBank

(a)

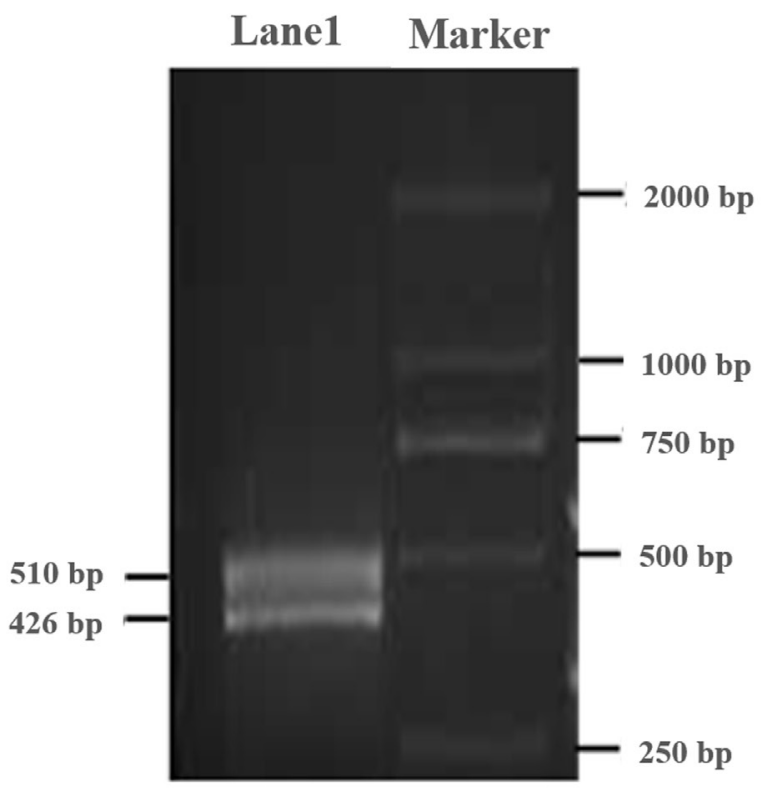

accession number: KY670835), which further proved that there were two alternative splice variants; no other variants were found in the pig muscle tissues. The CDS of the pig $M Y N N-1$ and $M Y N N-2$ variants is 1,830 bp and 1,746 bp respectively, MYNN-2 lacked the sixth exon than $M Y N N-1$, as shown in Supplementary Figure S1. Pig MYNN-1 CDS is composed of 1,830 bp encoding 609 amino acids, whereas MYNN-2 CDS is composed of 1,746 bp encoding 581 amino acids.

\section{Bioinformatics analysis}

The prediction of function domain found MYNN-1 contains an amino-terminal $\mathrm{BTB} / \mathrm{POZ}$ domain at the $\mathrm{N}$ end and eight tandem repeated zinc-finger motifs of the $\mathrm{C}_{2} \mathrm{H}_{2}$-type at the $\mathrm{C}$ end. Nevertheless, MYNN-2 also contains a BTB domain at the $\mathrm{N}$ end, and differs from MYNN-1 in that it less a zinc finger protein domain at the $\mathrm{C}$ end (Supplementary Figure S2).

MegAlign software was used to determine the homology of MYNN amino acid sequences of 10 species including the pig, human, and sheep. Phylogenetic trees (as shown in Fig-

(c)

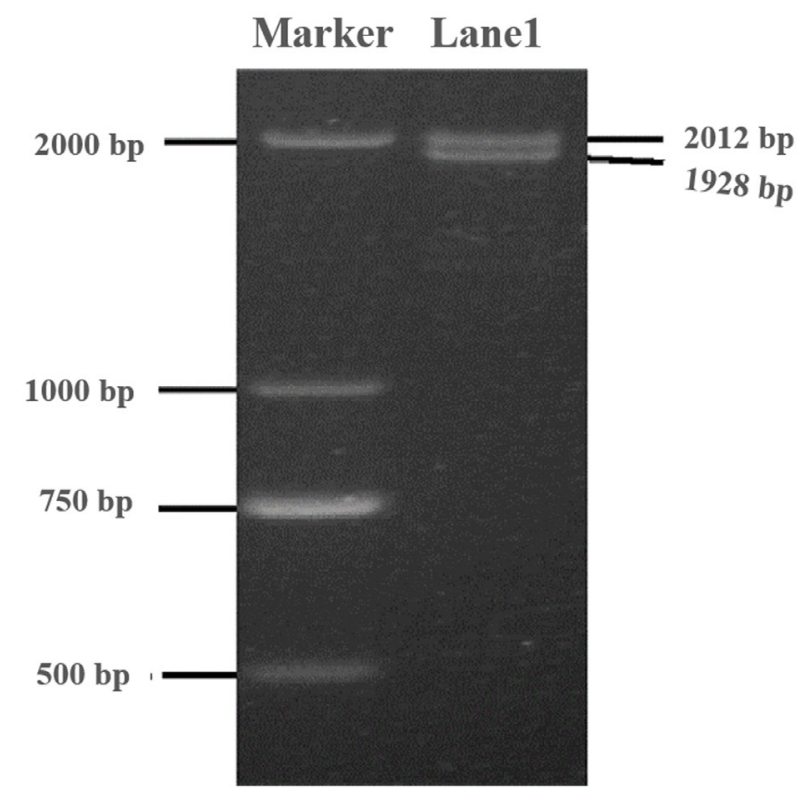

(b)

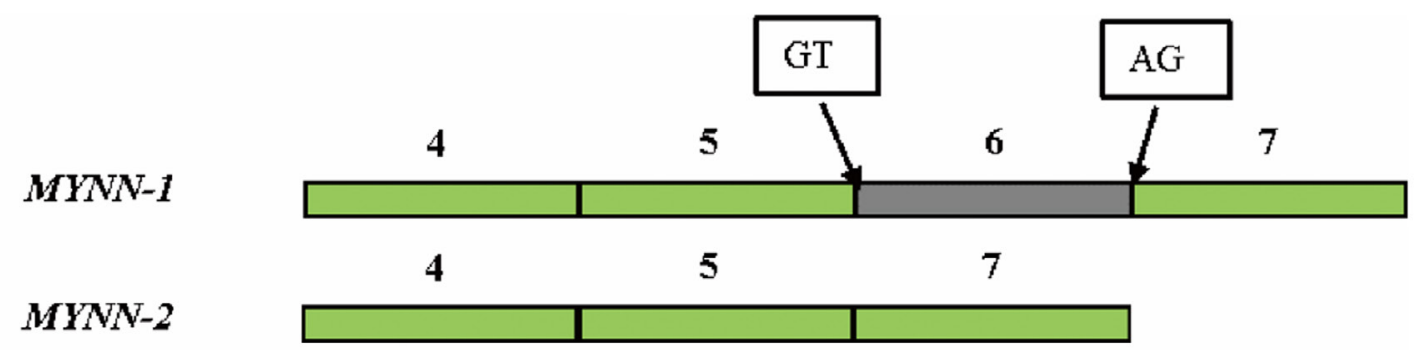

Figure 2. Products amplified by the P1 and P2 primers. (a) Products amplified by the P1 primer; (b) Coding sequence structure diagram of MYNN-1 and MYNN-2; (c) Products amplified by the P2 primer. MYNN, myoneurin. 
ure 3) of 10 species were constructed by the neighbor-joining method using Mage 7.0. The results showed that the MYNN1 amino acid sequence was $97 \%$ homologous with Bos Taurus isoform X1, Canis lupus familiaris isoform X1, Pantholops hodgsonii isoform X1 and Ovis aries isoform X1. The homology of Sus scrofa MYNN-1 amino acid sequence with Pantholops hodgsonii isoform X2, Sus scrofa MYNN-2, Oryctolagus cuniculus isoform X1, Homo sapiens isoform X1, Gorilla gorilla gorilla isoform X1 and Nomascus leucogenys isoform X1 was 96\%, 95\%, 95\%, 95\%, 95\%, and 95\%, respectively. The MYNN-2 amino acid sequence was most homologous with Canis lupus familiaris isoform X2 and Ovis aries isoform X2, with homology of $97 \%$. Mus musculus MYNN, as another branch, was distant with the other nine species. The MYNN development tree is consistent with the evolution of species, which suggests that MYNN exhibits similar functions in different species.

Tissue expression patterns of $M Y N N-1$ and $M Y N N-2$ $M Y N N-1$ and $M Y N N-2$ were widely distributed in all of the tissues, and there were significant differences in expression in different tissues (Figure 4). Notably, the two variants tended to have higher expression levels of ST, LU, and P in MS pig than in LW pig $(\mathrm{p}<0.01)$. Nevertheless, their expression levels in the CE, K, LI, and SP of LW pig were significantly higher than in the MS pig $(\mathrm{p}<0.01, \mathrm{p}<0.05)$; The relative expression of $M Y N N-1$ and $M Y N N-2$ was higher in CE, LI, K, and was lowest in F in LW pig. Meanwhile, their expression levels was higher in ST, P, SI, and was lowest in F in MS pig; The expression level of MYNN-1 was significantly higher than $M Y N N-2$ in almost tissues in both breeds $(\mathrm{p}<0.01, \mathrm{p}<0.05)$.

Developmental expression profile of $M Y N N-1$ and MYNN-2 in CE

During the growth and development of LW and MS pigs, $M Y N N-1$ and $M Y N N-2$ exhibited similar expression patterns in $\mathrm{CE}$ (Figure 5a, 5b). The two variants tended to have higher expression levels in MS pig than in LW pig at $1 \mathrm{~d}$ time point $(p<0.01)$. Nevertheless, their expression levels of LW pig were significantly higher than in MS pig at other time points $(\mathrm{p}<$ 0.01 ); in the LW pig, the expressions of MYNN-1 and MYNN2 increased gradually with the increase of age, whereas, the expressions of MYNN-1 and MYNN-2 decreased gradually in MS pig; MYNN-1 expression was significantly higher than that of MYNN-2 at different stages in each breed ( $\mathrm{p}<0.01, \mathrm{p}<$ 0.05).

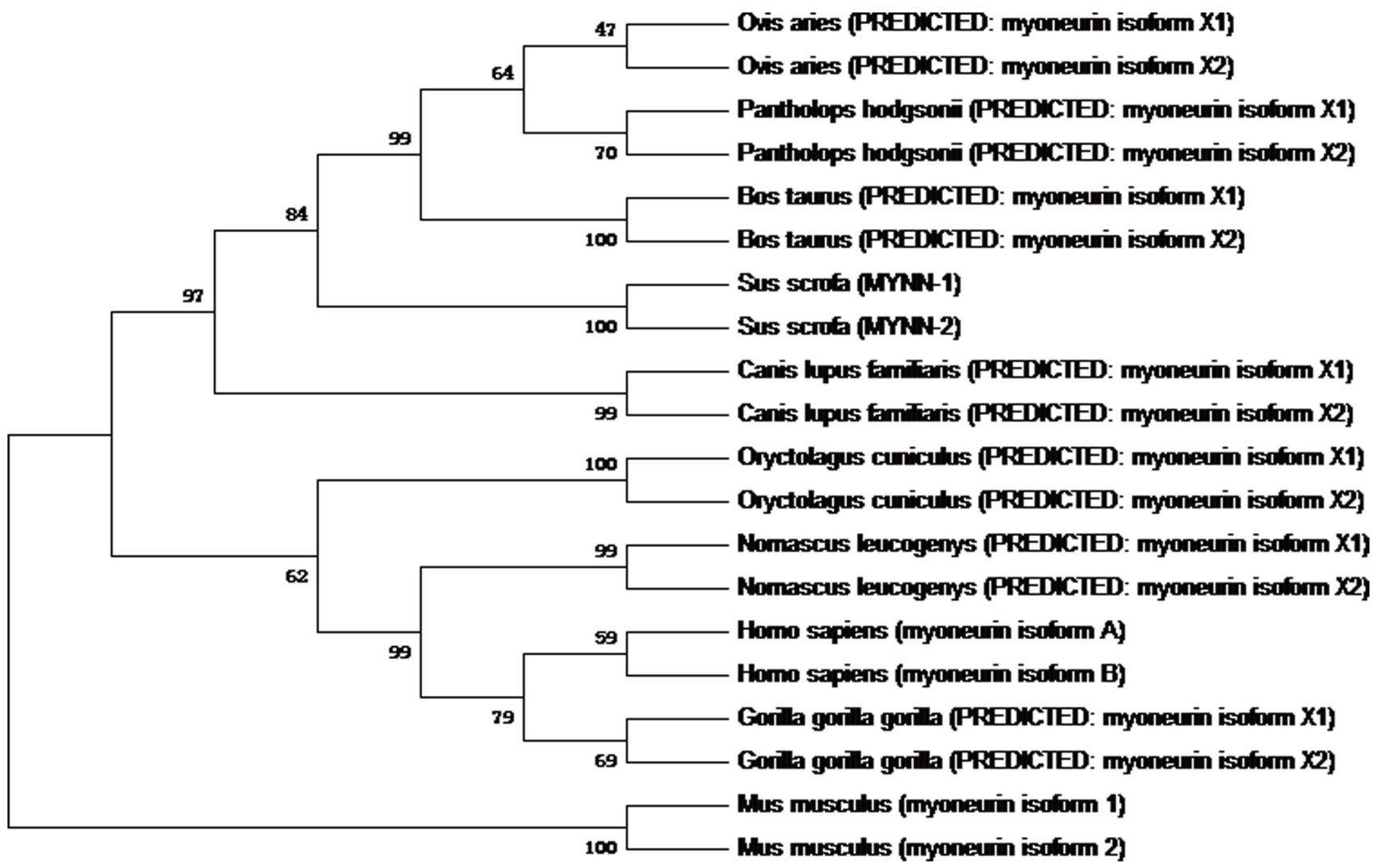

Figure 3. Phylogenetic tree of myoneurin (MYNN) amino acid sequences in 10 species. Ovis aries (XP_004003228.1; XP_011955094.1); Pantholops hodgsonii (XP_005964586.1; XP_005964587.1); Bos taurus (XP_005201749.1; XP_005201752.1); Canis lupus familiaris (XP_861907.2; XP_005639965.1); Oryctolagus cuniculus (XP_008264687.1; XP_002716433.1); Nomascus leucogenys (XP_012366261.1; XP_012366262.1); Homo sapiens (NP_001172047.1; NP_001172048.1); Gorilla gorilla gorilla (XP_004038031.1; XP_018880272.1); Mus musculus (NP_085034.2; NP_001276550.1). 
(a)
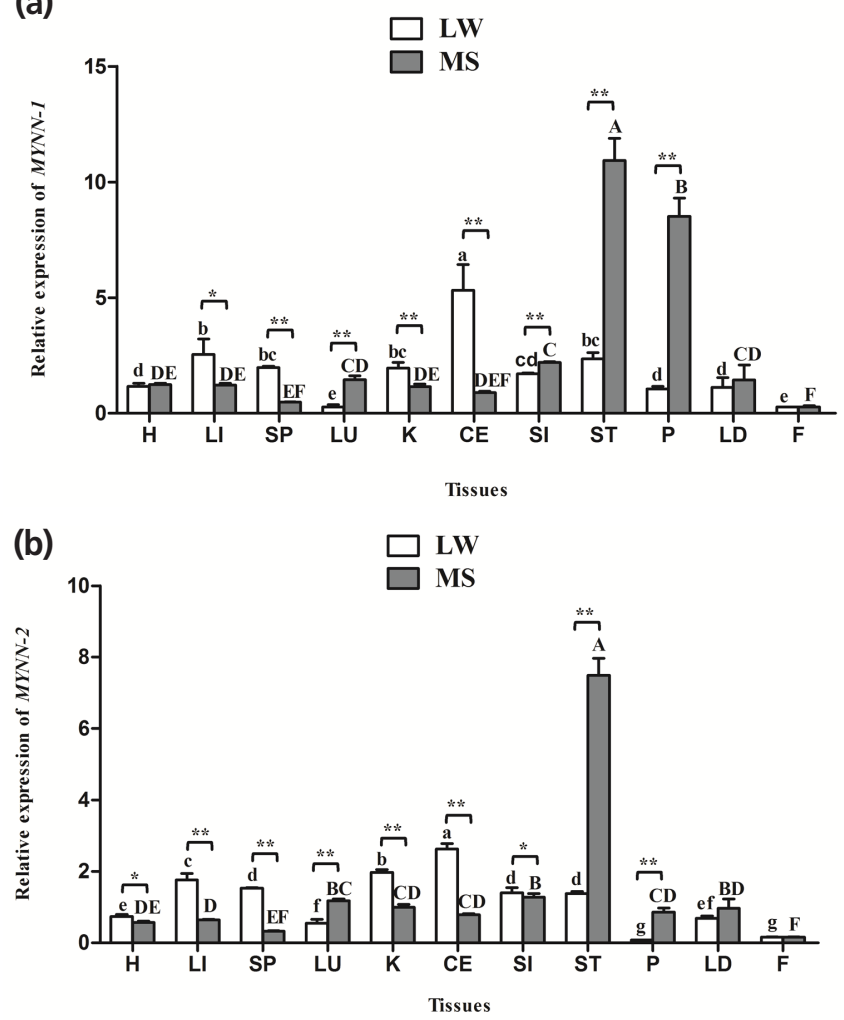

Figure 4. MYNN mRNA expression in pig tissues. (a) Relative mRNA expression of MYNN-1 in different tissues of LW and MS pigs; (b) Relative mRNA expression of MYNN-2 in different tissues of LW and MS pigs. MYNN, myoneurin; LW, Large White pig; MS, Mahsen pig; $H$, heart; LI, liver; SP, spleen; LU, lung; K, kidney; $C E$, cerebellum; SI, small intestine; ST, stomach; P, pancreas; LD, longissimus dorsi; F, fat. Values in the bar diagram indicate "mean \pm standard errors". In the same breed, means with no common letter differ significantly. In the same tissue, "*" indicates that the difference between LW and MS had a significance level of 0.05 , "**" indicates that the difference between LW and MS had a significance level of 0.01

\section{Developmental expression profile of $M Y N N-1$ and $M Y N N-2$ in ST}

In ST, the two variants tended to have higher expression levels in LW pig than in MS pig at $180 \mathrm{~d}$ time point $(\mathrm{p}<0.01)$ (Figure $5 c, 5 d)$. Nevertheless, their expression levels of LW pig were significantly higher than in MS pig at other time points $(\mathrm{p}<$ 0.01 ); In LW pig, the expressions of $M Y N N-1$ and $M Y N N-2$ increased gradually with the increase of age, whereas, the expressions of $M Y N N-1$ and $M Y N N-2$ decreased gradually in MS pig; $M Y N N-1$ expression was significantly higher than that of $M Y N N-2$ at almost stages in each breed $(\mathrm{p}<0.01)$.

\section{Developmental expression profile of $M Y N N-1$ and $M Y N N-2$ in LD}

In $\mathrm{LD}$, the two variants tended to have higher expression levels in MS pig than in LW pig at $1 \mathrm{~d}$ time point $(\mathrm{p}<0.01)$ (Figure $5 \mathrm{e}, 5 \mathrm{f})$; In the LW pig, the two variants were significantly more highly expressed in the $\mathrm{LD}$ at $1 \mathrm{~d}$, whereas, the expression was highest at $90 \mathrm{~d}$ in the MS pig; With the exception of the $90 \mathrm{~d}$ time point in MS pig, $M Y N N-1$ expression was significantly higher than that of $M Y N N-2$ at different stages in each breed $(\mathrm{p}<0.01, \mathrm{p}<0.05)$.

\section{DISCUSSION}

Alternative splicing refers to an pre-mRNA through different splicing, ultimately resulting in different structural and functional protein isoforms [26]. Alternative splicing is widespread in the body, and is related to changes in protein function [27]. Using a new generation of high-throughput sequencing technology, RNA-seq being used to discover and identify alternatively spliced events in animals and plants for which can accurately quantify gene expression in tissues and identify gene sequences accurately [28,29]. Mortazavi et al [30] detected 3,500 splicing variants in mice by RNA-seq, and found that there were 145,000 mRNA splicing forms. Graveley et al [29] detected multiple developmental stages of transcription in Drosophila melanogaster by RNA-seq technology. The results showed that 12,295 exons were found have 22,965 new splice sites, and more than half of them were variable splicing [31]. This experiment was based on the sequencing results of RNA-seq to study the alternative splicing of pig $M Y N N$ gene, and we obtained two novel alternative isoforms of pig MYNN for the first time. MYNN-1 CDS is composed of 1,830 bp encoding 609 amino acids, $M Y N N-2$ CDS is composed of 1,746 bp encoding 581 amino acids.

The expression levels of each transcript of the same gene is different, and the transcript with highest expression is known as the main isoform, whereas those with relatively few transcripts are called secondary isoforms [32]. In our study, the mRNA expression level of $M Y N N-1$ was significantly higher than MYNN-2 in most tissues ( $\mathrm{p}<0.01, \mathrm{p}<0.05)$, meanwhile, the expression of $M Y N N-1$ in the CE, ST, LD were significantly higher than $M Y N N-2$ at most stages. Thus, it is clear that $M Y N N-1$ is the main variant in pig $(\mathrm{p}<0.01, \mathrm{p}<0.05)$.

Differences in gene expression are closely related to physiological functions, so evaluation of gene expression characteristics provided the basis for studying the function of $M Y N N-1$ and $M Y N N-2$ genes. Previous studies have found that BTB/POZ protein is widely expressed in various tissues of mammals [2022]. In humans, the expression of a $2.5 \mathrm{~kb}$ MYNN was found to be more highly expressed in muscle than in other tissues, and was detected in the cerebellum, skeletal muscle, testis, hearth, brain, and LI tissues of the mouse [17]. The results of our study showed that MYNN-1 and MYNN-2 were ubiquitously but differentially expressed in the H, LI, SP, LU, K, CE, SI, ST, P, $\mathrm{LD}$, and $\mathrm{F}$, which were basically consistent with previous studies $(p<0.05)$. The universal expression of the MYNN gene is consistent with its function as a transcription factor to regulate gene transcription. Further analysis showed that MYNN-1 and 
(a) $\square$ LW
$\square$ MS

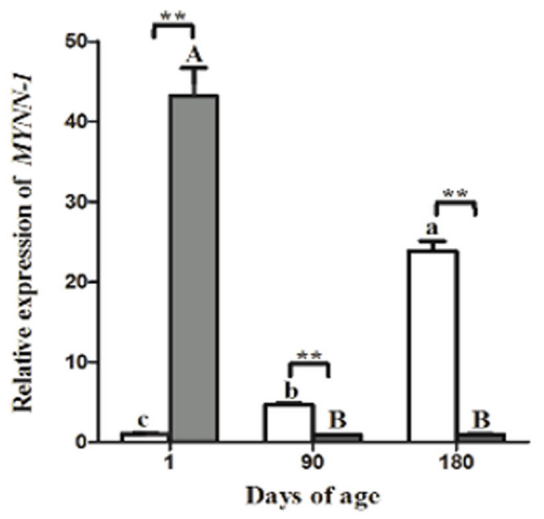

(c)

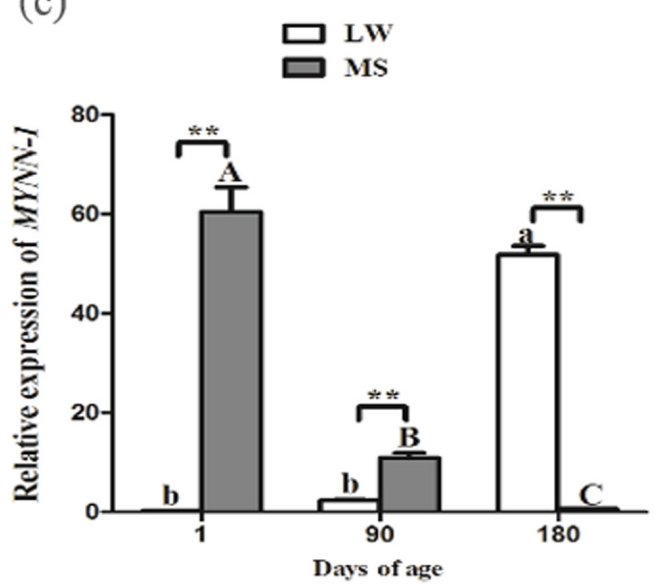

(e)

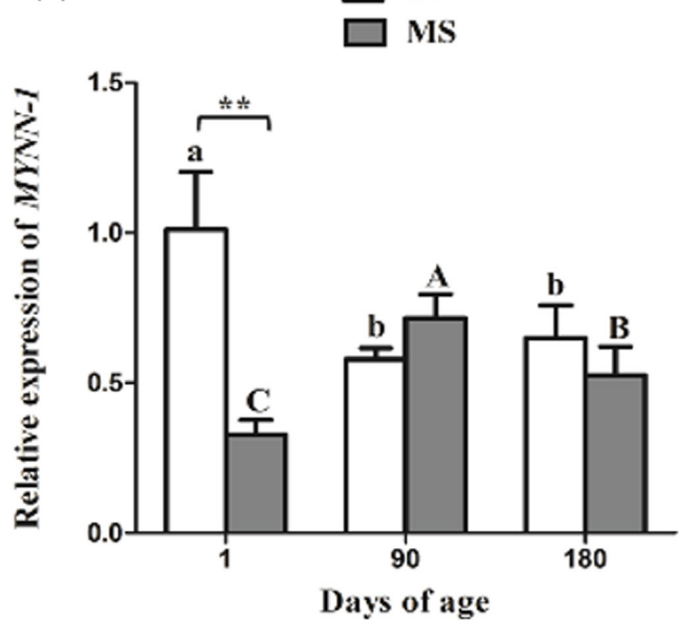

(b) $\square$ LW $\square$ MS

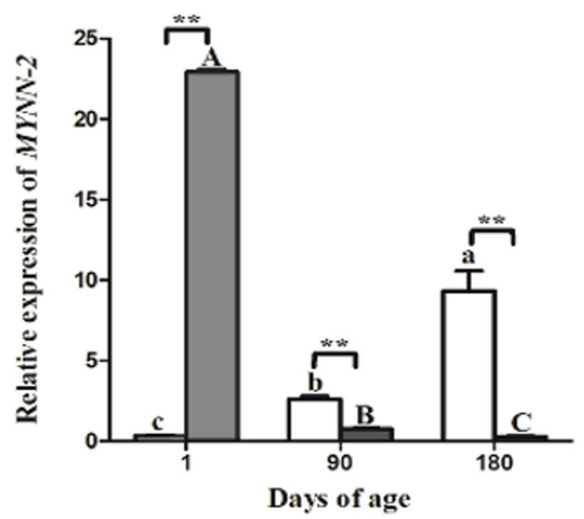

(d)

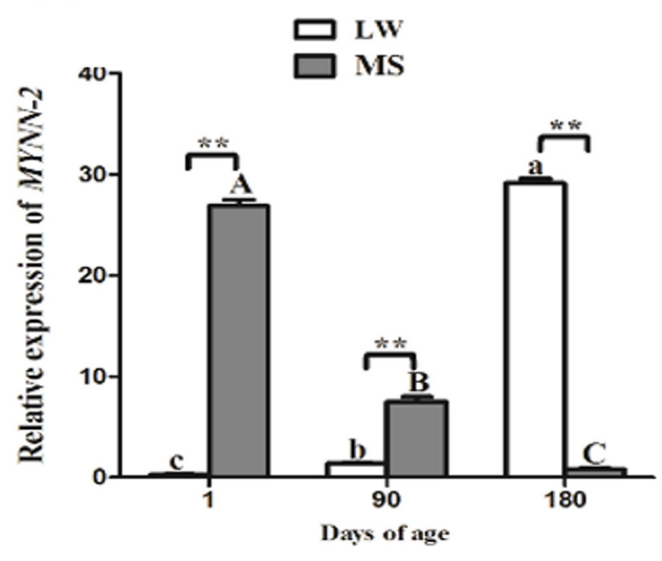

(f) $\square$ LW

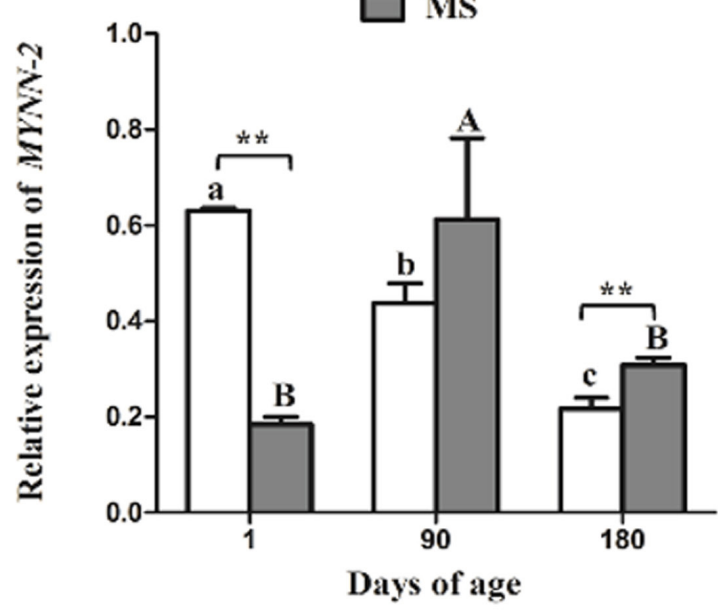

Figure 5. MYNN-1 and MYNN-2 expression in CE, ST, and LD of LW and MS pigs at different time points. (a) Relative mRNA expression of MYNN-1 in LW and MS pigs at different stages; (b) Relative mRNA expression of MYNN-2 in LW and MS pigs at different stages; (c) Relative mRNA expression of MYNN-1 in LW and MS pigs at different stages; (d) Relative mRNA expression of MYNN-2 in LW and MS pigs at different stages; (e) Relative mRNA expression of MYNN-1 in LW and MS pigs at different stages; ( $f$ ) Relative mRNA expression of MYNN-2 in LW and MS pigs at different stages. MYNN, myoneurin; CE, cerebellum; ST, stomach; LD, longissimus dorsi; LW, Large White pig; MS, Mahsen pig. In the same breed, means with no common letter differ significantly. In the same tissue, ${ }^{\prime * *}$ " indicates that the difference between LW and MS had a significance level of 0.01 . 
MYNN-2 were highest expressed in CE in LW pig, which proved that MYNN may relate to the development of CE. Those two isoforms were highly expressed in the ST, P, and SI of MS pig, and the ST, P, and SI all are main digestive organs. Therefore, we hypothesized that MYNN is closely related to the digestion and absorption of MS pig. In addition, these two isoforms of different expression characteristics in different breeds may be caused by the different genetic background of LW pig and MS pig.

Therefore, we further studied the developmental expression profile of $M Y N N-1$ and $M Y N N-2$ in ST, the result showed that: The expression of MYNN-1 and MYNN-2 decreased gradually with the increase of age in MS pig. The gastrointestinal tract are not fully developed when piglets was born. Its weight and volume were relatively small, the secretion of digestive enzymes was insufficient, the digestion and absorption function was not complete, such as pepsin exists in the state of zymogen in piglet birth, which can not digest protein, especially vegetable protein. The piglets at 1 day have a ST weight of only 4 to $8 \mathrm{~g}$, which can hold only about $40 \mathrm{~mL}$ of milk. The gastrointestinal tract of piglets developed rapidly during the whole lactation period, and pepsin began to have digestive function at 35 to 40 days. The digestive function gradually completed at 75 days old, and the gastrointestinal tract was no longer developed at 180 days. It indicted that the developmental expression profile of $M Y N N-1$ and MYNN-2 in ST were consistent with gastrointestinal tract in MS pig. Nevertheless, for the LW pig, these two isoforms showed a downward trend with the increase of the age, so we infered LW pig MYNN had the opposite effect with MS pig.

By analyzing the overall structure of MYNN and examining its expression in human muscle, Alliel et al [33] showed that MYNN can regulate gene expression in the muscle. In our study, MYNN-1 and MYNN-2 had lowest expression in F and moderate expression in LD compared with other tissues in both LW and MS pigs. Therefore, to further study the expression patterns of MYNN in muscle tissue, we examined the mRNA expression of MYNN-1 and MYNN-2 in different developmental stages in both breeds. Interestingly, the gene expression trends of $M Y N N-1$ and $M Y N N-2$ were similar in the splice variants. $M Y N N-1$ and $M Y N N-2$ were most highly expressed in the LD at $1 \mathrm{~d}$ compared to other stages in the LW pig. It is generally believed that the number of muscle fibers will no longer change after the birth of an animal, and that the muscle growth depends mainly on the thickening of the muscle fiber rather than the increase in the number [33]. The development of muscle fiber basically concentrated in the period, which from pregnancy until 2 month old after birth of two months, suggesting MYNN has the same development trend as skeletal muscle fiber. The expression of $M Y N N-1$ and $M Y N N-2$ increased first and then decreased with the increase of age for MS pig. The development of skeletal muscle is regulated by a series of myogenic determinants. The myogenic determining factor $(M y o D)$ and myogenin $(M y o G)$ genes were belong to the myogenic regulatory family, which is involved in the formation of muscle fiber and is a candidate gene for muscle growth and meat quality [34]. Our research group previous studied and found that $M y o D$ showed the trend of first rising and then decreasing at the 1 to 180 days of age, and the expression of $M y o G$ was significantly lower at 1 and 180 days, and reached the highest value at 90 days [34].The developmental expression profile of $M Y N N-1$ and $M Y N N-2$ were the basically same with those two myogenic factor.

\section{CONCLUSION}

In this paper, two transcripts of $M Y N N$ (MYNN-1 and $M Y N N$ 2) were identified in muscle tissue of pigs for the first time. And $M Y N N-1$ was main variant. MYNN was highly expressed in ST, CE, and LD, and their expression were regular. We speculated that MYNN played important roles in digestion/ absorption and skeletal muscle growth, whereas the specific mechanisms were still remaining to be further elucidated.

\section{CONFLICT OF INTEREST}

We certify that there is no conflict of interest with any financial organization regarding the material discussed in the manuscript.

\section{ACKNOWLEDGMENTS}

We thank LetPub (www.letpub.com) for its linguistic assistance during the preparation of this manuscript. Thanks to all the staff of the Datong pig farm. This work was supported by the Program for Sanjin Scholar, the Fund for Shanxi 1331 Project and the Foundation of Science and Technology Innovation Team of Shanxi Province (grants No. 201705D13102819).

\section{REFERENCES}

1. Aravind L, Koonin EV. Fold prediction and evolutionary analysis of the POZ domain: structural and evolutionary relationship with the potassium channel tetramerization domain. J Mol Biol 1999;285:1353-61.

2. Li X, Peng H, Schultz DC, et al. Structure-function studies of the $\mathrm{BTB} / \mathrm{POZ}$ transcriptional repression domain from the promyelocytic leukemia zinc finger oncoprotein. Cancer Res 1999;59:5275-82.

3. Takenaga M, Hatano M, Takamori M, et al. Bcl6-dependent transcriptional repression by BAZF. Biochem Biophys Res Commun 2003;303:600-8.

4. Phan RT, Dalla-Favera R. The BCL6 proto-oncogene suppresses 
p53 expression in germinal-centre B cells. Nature 2004;432: 635-9.

5. Kelly KF, Otchere AA, Graham M, Daniel JM. Nuclear import of the BTB/POZ transcriptional regulator Kaiso. J Cell Sci 2004;117:6143-52.

6. Huynh KD, Bardwell VJ. The BCL-6 POZ domain and other POZ domains interact with the co-repressors $\mathrm{N}-\mathrm{CoR}$ and SMRT. Oncogene 1998;17:2473-84.

7. Prag S, Collett GD, Adams JC. Molecular analysis of muskelin identifies a conserved discoidin-like domain that contributes to protein self-association. Biochem J 2004;381:547-59.

8. Li X, Zhang D, Hannink M, Beamer LJ. Crystal structure of the Kelch domain of human Keap1. J Biol Chem 2004;279: 54750-8.

9. Ledee DR, Gao CY, Seth R, et al. A specific interaction between muskelin and the cyclin-dependent kinase 5 activator p39 promotes peripheral localization of muskelin. J Biol Chem 2005;280:21376-83.

10. Melnick A, Carlile G, Ahmad KF, et al. Critical residues within the BTB domain of PLZF and Bcl- 6 modulate interaction with corepressors. Mol Cell Biol 2002;22:1804-18.

11. David G, Alland L, Hong SH, et al. Histone deacetylase associated with $\mathrm{mSin} 3 \mathrm{~A}$ mediates repression by the acute promyelocytic leukemia-associated PLZF protein. Oncogene 1998;16: 2549-56.

12. Ahmad KF, Melnick A, Lax S, et al. Mechanism of SMRT corepressor recruitment by the BCL6 BTB domain. Mol Cell 2003;12:1551-64.

13.Zhou C, Li LY. Advances in $\mathrm{C} 2 \mathrm{H} 2$ zinc finger proteins. Life Sci Res 2004;8:215-20.

14. Cifuentes-Diaz C, Bitoun M, Goudou D, et al. Neuromuscular expression of the $\mathrm{BTB} / \mathrm{POZ}$ and zinc finger protein myoneurin. Muscle Nerve 2004;29:59-65.

15. Alliel PM, Seddiqi N, Goudou D, et al. Myoneurin, a novel member of the BTB/POZ-zinc finger family highly expressed in human muscle. Biochem Biophys Res Commun 2000;273: 385-91.

16. Alliel PM, Cifuentès-Diaz C, Bitoun M, et al. Expression of the transcriptional repressor gene myoneurin at the neuromuscular junction, during mouse development and in the adult. J Physiol Paris 2006;99:1.

17. Meng YY, Xu XZ, Liao J. ZBTB transcription factors and tumorigenesis. Life Sci 2015;27:477-85.

18. Trappe R, Buddenberg P, Uedelhoven J, et al. The murine BTB/ POZ zinc finger gene Znf131: predominant expression in the developing central nervous system, in adult brain, testis, and thymus. Biochem Biophys Res Commun 2002;296:319-27.

19. Sakashita C, Fukuda T, Okabe S, et al. Cloning and characterization of the human BAZF gene, a homologue of the BCL6 oncogene. Biochem Biophys Res Commun 2002;291:567-73.

20. Mitchelmore C, Kjaerulff KM, Pedersen HC, et al. Characterization of two novel nuclear BTB/POZ domain zinc finger isoforms. Association with differentiation of hippocampal neurons, cerebellar granule cells, and macroglia. J Biol Chem 2002;277:7598-609.

21.Zhao YY, Gao PF, Li W, et al. Study on the developmental expression of Lbx1 gene in longissimus dorsi of Mashen and Large White Pigs. Italian J Anim Sci 2015;14:2081-7.

22.Zhang J. Species diversity and the way to protect MA SHEN ZHU. Shanxi, China: J Shanxi Agric Univ; 2001.

23. Yang WP, Cao GQ, Shi JZ, Liu JH, Zhou ZX. Study on the finishing ability of different cross combination in pig. Chinese J Anim Sci 2005;41:48-9.

24. Kim D, Pertea G, Trapnell C, et al. TopHat2: accurate alignment of transcriptomes in the presence of insertions, deletions and gene fusions. Genome Biol 2013;14:R36.

25. Ghosh S, Chan CK. Analysis of RNA-Seq data using TopHat and Cufflinks. Methods Mol Biol 2016;1374:339-61.

26.Zhang XN, Xiao HS. Progress on the method of identification of alternative splicing. Life Science Instruments; 2008.

27. Modrek B, Lee C. A genomic view of alternative splicing. Nat Genet 2002;30:13-9.

28. Marquez Y, Brown JW, Simpson C, Barta A, Kalyna M. Transcriptome survey reveals increased complexity of the alternative splicing landscape in Arabidopsis. Genome Res 2012;22:118495.

29. Graveley BR, Brooks AN, Carlson JW, et al. The developmental transcriptome of Drosophila melanogaster. Nature 2011;471: 473-9.

30. Mortazavi A, Williams BA, McCue K, Schaeffer L, Wold B. Mapping and quantifying mammalian transcriptomes by RNA-Seq. Nat Methods 2008;5:621-8.

31. Trapnell C, Pachter L, Salzberg SL. TopHat: discovering splice junctions with RNA-Seq. Bioinformatics 2009;25:1105-11.

32.Feng H, Qin Z, Zhang X. Opportunities and methods for studying alternative splicing in cancer with RNA-Seq. Cancer Lett 2013;340:179-91.

33. Ashmore CR, Addis PB, Doerr L. Development of muscle fibers in the fetal pig. J Anim Sci 1973;36:1088-93.

34. Niu JY. Study on the developmental expression of Pax7, MyoD and MyoG genes in longissimus dorsi of pigs. Shanxi, China: Shanxi Agricultural University; 2015. 